\title{
Endoscopy Room Behavior
}

\section{Introduction}

"Don't bring food and drinks inside!"We all know that this is one of the library's rules, what you should know is that you must apply the same in the Endoscopy room beside other rules such as don't speak loudly.

Have you ever heard ofa ship with two captains?! In the endoscopy room the physician is the only captain who takes all responsibility of the patient in terms of safety and convenience. Although safety is important for all staff members who are involved in theprocedure; starting from cleaning and disinfecting the scopes before every procedure, to assisting the physician during the procedure and finally cleaning and disinfecting the scope again for storage area. Before the procedure the technician must follow the standard guidance for protection of the disinfection process. Dealing with contamination items is like dealing with a time bomb! Believe me the right outfit and protection really matter for your own safety and I'm sure nobody will hesitate to consider that.

Preparation of the procedureis really important for both the patient and the endoscopy room. The patient needs to be mentally and physically prepared. For sure it is difficult for some people to imagine that a big tube will be inserted into their body from up or down. Most doctors already are doing a great job talking to the patient before the procedure, giving a brief image of what the procedure is and clarifying the status before and after the procedures and that's the mental part. As for the Physical preparation part, the doctorinstructs the patient with the necessary steps that must be followed according to the procedure type (EX: gastro or colonoscopy). In addition, the assisting nurse must carefully observe and guide the patient during the preparation process to ensure that he indeed followed the doctor's instructions and that's the physical part. Once the patient is mentally and physically ready, procedure should go smoothly without any complications.

During the endoscopy procedure the physician AKA the captain of the room does the main part while staying in touch with the patient, giving him details about the progress of the procedure, this will help toreassure the patient. Incidents where staff yellin the endoscopy room during a procedure makes the patient feel that his case is critical and thus builds up tension in the room. The assistant must realize that the current patient is not the same patient of yesterday or the day before. Keep in mind that this might be totally new to many patients, therefore avoid any irrelevant conversations in the endoscopy room in the presence of the patient, instead talk about the patient and about his procedure,this will give him the impression that he is the center of attention and that everyonepresent in the room really cares about his or her convenience. Don't try to sing if you really aren't good at it because nothing is more offensive for the patient than a big tube in his behind. You also have to be very picky with your expressions about what you see on the monitor of the endoscopy machine, don't overreactwith "oh my god", "ouch" or "what's that"! Those kinds of words are really enough to make a patient turn suddenly to check what's going on and what you found inside him.

Make sure that you don't run, walk or walk fast, this is vital for you, your colleagues, doctors and the patient to avoid traffic in the
Volume 5 Issue 4 - 2016

\author{
Alaa Ahmed* \\ Biomedical \& Systems Engineering, Netherlands \\ Correspondence: Ala Ahmed, Biomedical \& Systems \\ Engineering, Ortler 99, Netherlands, \\ Email alaa.ahmed@ieeeypegypt.org \\ Received: October 31, 2016 | Published: November 07, 2016
}

Endoscopy room. Some points you really need to consider if you want to give good assistance to the physician; firstly, consider if the physician is right or left handed, this will make a huge difference in terms of the setting inside the room especially the endoscopy tower, the patient table and if you are using an external mounted monitor.

Secondly, try as much as you can to keep the room with suitable space of moving; cables and wires should be spread in a seen location. Also when you have visitors or company representatives they will know where to stand during the procedures and if they don't know, you have to guide them for a good location.

Thirdly, regarding the gastro procedure the assistant must always be aware about the mouthpiece because you really don't want the patient to bite the scope, harm himself with scope fractures,or failure of lubricate which can lead to patient discomfort and trauma in his upper or lower part of.

As for company representatives, if they present during the endoscopy procedure for technical advice or equipment applications, the easiest way to try new techniques is by performing it first on a mannequin or a free demonstration so that both the doctor and assistant can build an image about how it works, and then apply it on the patient. Last but not least is the prevention of infection inside the endoscopy room for the patient and endoscopy team. Everyone involved in the procedure is responsible for reducing the possibility of risk of instruments and equipment contamination; therefore you have to scrub the fit protections (gloves, goggles and facemasks) in the endoscopy room, as well as in the disinfection and cleaning area.

Don't forget to deliver the patient with a comfort face to the recovery area and make sure to assure him or her after the procedure.

Always keep the good work inside and around the endoscopy room. 